University of Louisville

ThinkIR: The University of Louisville's Institutional Repository

Faculty Scholarship

$5-2014$

Interleukin-6 and soluble interleukin-6 receptor levels in

posttraumatic stress disorder : associations with lifetime diagnostic status and psychological context.

\author{
Tamara L. Newton \\ University of Louisville \\ Rafael Fernandez-Botran \\ University of Louisville \\ James J. Miller \\ University of Louisville \\ Vicki Ellison Burns \\ University of Louisville
}

Follow this and additional works at: https://ir.library.louisville.edu/faculty

Part of the Psychology Commons

Original Publication Information

Newton, Tamara L., Rafael Fernandez-Botran, James J. Miller and Vicki Ellison Burns. "Interleukin-6 and Soluble Interleukin-6 Receptor Levels in Posttraumatic Stress Disorder: Associations with Lifetime Diagnostic Status and Psychological Context." 2014. Biological Psychology 99: 150-159.

This Article is brought to you for free and open access by ThinkIR: The University of Louisville's Institutional Repository. It has been accepted for inclusion in Faculty Scholarship by an authorized administrator of ThinkIR: The University of Louisville's Institutional Repository. For more information, please contact thinkir@louisville.edu. 


\title{
Interleukin-6 and Soluble Interleukin-6 Receptor Levels in Posttraumatic Stress Disorder: Associations with Lifetime Diagnostic Status and Psychological Context
}

\author{
Tamara L. Newton ${ }^{1}$, Rafael Fernandez-Botran², James J. Miller ${ }^{2}$, and Vicki Ellison Burns ${ }^{3}$ \\ ${ }^{1}$ Department of Psychological and Brain Sciences, University of Louisville \\ ${ }^{2}$ Department of Pathology and Laboratory Medicine, University of Louisville \\ ${ }^{3}$ School of Nursing, University of Louisville
}

\begin{abstract}
This study correlated lifetime PTSD diagnostic status with interleukin-6 (IL-6) and soluble IL-6 receptor (sIL-6R) levels, and tested whether these correlations are sensitive to psychological context. Midlife women attended two research visits where blood was drawn (beginning of visits) and saliva and oral mucosal transudate were collected (beginning and end of visits) to measure IL-6 and sIL-6R. Women were classified as PTSD-/- (past and current symptoms below subsyndromal levels), PTSD+/- (past symptoms at or above subsyndromal levels), or PTSD +/+ (past and current symptoms at or above subsyndromal levels). PTSD+/+ women, compared to the other women, showed more negative emotion at the beginning of the visits, higher salivary IL-6 levels at the beginning versus end of visits, and positive correlations between negative emotion, salivary IL-6, and plasma sIL-6R. Their plasma sIL-6R levels exceeded those of the PTSD+/women. Overall, IL-6 sensitivity to anticipation and to negative emotions, and higher sIL-6R levels, differentiated persistent versus remitted PTSD.
\end{abstract}

\section{Keywords}

PTSD; remitted PTSD; inflammation; IL-6; sIL-6R; anticipation

\begin{abstract}
In the early aftermath of traumatic stress exposure, a symptom profile consistent with posttraumatic stress disorder (PTSD) can be observed in up to three-quarters of persons, depending on the type of trauma (Riggs, Rothbaum, \& Foa, 1995). Still, not all traumaexposed persons show this symptom profile, and among those who do, there are diverse
\end{abstract}

(C) 2014 Elsevier B.V. All rights reserved.

Address correspondence to: Tamara L. Newton, Department of Psychological and Brain Sciences, University of Louisville, 317 Life Sciences, Louisville KY 40292. tlnewton@louisville.edu.

Vicki Ellison Burns is now at the Lansing School of Nursing and Health Sciences, Bellarmine University.

Conflict of Interest Statement: All authors declare that there are no conflicts of interest.

Publisher's Disclaimer: This is a PDF file of an unedited manuscript that has been accepted for publication. As a service to our customers we are providing this early version of the manuscript. The manuscript will undergo copyediting, typesetting, and review of the resulting proof before it is published in its final citable form. Please note that during the production process errors may be discovered which could affect the content, and all legal disclaimers that apply to the journal pertain. 
symptom trajectories, with some persons developing persistent, chronic PTSD, and others showing symptom remission with the passage of time following the traumatic event (Berntsen et al., 2012; Bonanno, Galea, Bucciarelli, \& Vlahov, 2006; Hobfoll et al., 2009; Layne, Warren, Watson, \& Shalev, 2007). Building on our previous research concerning PTSD symptoms and markers of inflammation (Newton et al., 2013), the present study examined how classifying PTSD status by considering both past and current symptoms affects associations with levels of the cytokine interleukin-6 (IL-6). We extended our studies to IL-6 measured in oral fluids (i.e., saliva and oral mucosal transudate), to the soluble IL-6 receptor (sIL-6R), and to consideration of the hypothesis that connections between IL-6 and lifetime PTSD status may be dynamic, or sensitive to psychological context.

Potential connections between PTSD and cytokine levels, particularly those that promote inflammation, have recently received much attention. This has been partially motivated by correlations between PTSD and elevated risk of chronic diseases with inflammatory pathophysiology (Ahmadi et al., 2011), and the question of whether alterations in cytokine levels-inflammatory mediators that are also stress-reactive-might help explain this connection. Generally speaking, across different types of traumatic stressors (e.g., accidents, war zone exposure, civilian assault), across different proinflammatory cytokines (e.g., IL-1 $\beta$, TNF-a, IL-6), and across both circulating and stimulated cytokine levels, evidence for a proinflammatory profile of PTSD and its symptoms is observed, although null and contrary evidence are also apparent (Gill, Saligan, Woods, \& Page, 2009; Pace \& Heim, 2011; Wong, 2002).

Of interest in the present study are connections between PTSD and the cytokine IL-6. In addition to being a predictor of chronic diseases itself (Ridker, Hennekens, Buring, \& Rifai, 2000), IL-6 has proinflammatory properties that include stimulating synthesis of C-reactive protein, a widely used indicator of systemic inflammation (Gabay \& Kushner, 1999; Ridker, 2003). Supporting a proinflammatory model of PTSD, the results of some studies have shown that circulating IL-6 levels are greater among persons with a current PTSD diagnosis compared to healthy controls (Maes et al., 1999) or trauma-exposed persons without PTSD (von Känel et al., 2010). Other studies, however, have either shown null effects (McCanlies et al., 2011; Vidovic et al., 2011), or elevations of IL-6 levels only when PTSD is comorbid with major depressive disorder (Gill, Luckenbaugh, Charney, \& Vythilingam, 2010). Thus, a clear picture of the connections between PTSD and IL-6 has not yet emerged.

In a previous report by our group, analyzing the association of PTSD symptom severity with markers of inflammation in a sample of midlife women, plasma IL-6 levels measured at an initial, baseline research visit were significantly, negatively correlated with past, but not current, symptom severity (Newton et al., 2013). This observation shows that PTSD symptom history has significance for current IL-6 levels, but because the direction of the correlation is negative, it appears to be at odds with the proinflammatory model of PTSD. One way to reconcile this may be to consider that for some persons, but not all, past symptoms subside. Thus, perhaps lower plasma IL-6 levels are linked with decreases in symptom severity, a pattern that could be revealed only by classifying participants using both past and current symptoms, rather than considering them separately. 
To test this idea, the present study identified three groups of women: PTSD-/- (no evidence of past or current syndromal or partial/subthreshold PTSD); PTSD+/- (presence of past, but not current, syndromal or partial/subthreshold PTSD); and PTSD+/+ (presence of past and current syndromal or partial/subthreshold PTSD). We specifically aimed to clarify the prior observation that severe past symptoms predicted lower IL-6 levels (Newton et al., 2013) by examining whether the PTSD+/- group_ persons previously, but no longer, reporting syndromal or subsyndromal symptoms - showed lower IL-6 levels than the PTSD+/+ group. The main goal was to examine whether these groups differed in terms of IL-6 levels in two oral fluids: saliva, a product of salivary glands, and oral mucosal transudate (OMT), fluid that has filtered through the oral mucosa from systemic sources (i.e., serum or plasma) (Nishanian, Azia, Chung, Detels, \& Fahey, 1998). However, we also included plasma IL-6 in order to more directly compare this group-based analytic approach to one using continuous symptom scores, as in our previous study. Levels of IL-6 from these three different fluids all show correlations with various psychosocial factors (Chiang, Eisenberger, Seeman, \& Taylor, 2012; Sjogren, Leanderson, Kristenson, \& Ernerudh, 2006), suggesting central nervous system control, but their intercorrelations are small to moderate (FernandezBotran et al., 2011; Sjogren et al., 2006), perhaps reflecting the somewhat different origins of IL-6 in each of the fluids. For example, as we previously showed, levels of IL-6 (and also its soluble receptor, sIL-6R) tend to be higher in OMT than in saliva and plasma, suggesting production by the oral mucosa (Fernandez-Botran et al., 2011)

As a second goal, the present study aimed to clarify another pattern observed in our prior report: severe past PTSD symptoms predicted higher plasma IL-6 levels at a visit that included a trauma assessment, compared to an initial baseline visit that did not include any trauma-related questions. Further, the blood used for IL-6 assays had been drawn at the beginning, rather than the end, of each research visit, implying that this response was anticipatory to confronting trauma-related content. In contrast, when past PTSD symptoms were reported as absent or low, plasma IL-6 levels were higher at the initial baseline visit compared to the trauma assessment visit, suggesting an anticipatory response to the novelty or unfamiliarity of the first visit (Al'Absi \& Lovallo, 1993; Ohman, Hamm, \& Hugdahl, 2000). These results further emphasize the importance of assessing PTSD symptom history, and also suggest that connections between PTSD symptoms and IL-6 levels may be dynamic, or sensitive to psychological context, and especially to anticipation. In the present study, we further evaluated this by taking advantage of the fact that oral fluids were collected at the beginning and at the end of both research visits. A pattern in which correlations between PTSD and IL-6 levels are higher at the beginning of a visit than at the end underscores the role of anticipation, compared to a pattern in which correlations are similar at both assessments (suggesting a static relationship, or a visit effect), or one in which correlations are higher at the end of a visit compared to the beginning (suggesting a reactivity response).

A third goal of the present study was to examine whether the predicted associations also extend to sIL-6R, endogenous regulators of the bioavailability and cellular targets of IL-6 (Heaney \& Golde, 1996; Rose-John, Scheller, Elson, \& Jones, 2006; Scheller, Chalaris, Schmidt-Arras, \& Rose-John, 2011). The few studies that have examined connections 
between sIL-6R and trauma-related symptoms have revealed positive correlations between serum sIL-6R levels and post-trauma psychological distress (Sutherland, Alexander, \& Hutchison, 2003), PTSD symptoms (Miller, Sutherland, Hutchison, \& Alexander, 2001), and syndromal PTSD, especially when comorbid with major depression (Maes et al., 1999). Thus, available evidence predicts positive associations between sIL-6R levels and PTSD, but does not provide any basis for predictions with respect to psychological context.

\section{Method}

\section{Participant Recruitment and Selection}

Physically healthy women with histories of divorce or separation were recruited from the community as part of a broader investigation of recovery from intimate partner violence (IPV) and inflammation at midlife (Newton et al., 2011). A phone interview assessed inclusion criteria (history of divorce; 45 to 60 years of age; post-menopausal defined as cessation of menses for $\geq 12$ months), and exclusion criteria (no English language skills; ongoing divorce-related legal issues; psychiatric hospitalization in the preceding 6 months; active suicidal ideation; current IPV-i.e., IPV involving an ex-partner in the preceding year, or any IPV history with a current partner, defined by a score $\geq 1$ on the Slapped, Threatened and Throw screener (Paranjape, Rask, \& Liebschutz, 2006); chronic disease other than unmedicated hypertension; use of prescription or over-the-counter medications with inflammatory effects (including psychotropics and botanicals); blood or needle phobia; use of street drugs; current alcohol use disorder defined as $\geq 5$ on the Alcohol Use Disorders Identification Test consumption questions (Dawson, Grant, Stinson, \& Zhou, 2005)).

Of 577 callers, 96 were eligible and interested in participation; 82 attended the first research visit. This visit included a blood draw and urine sample for additional eligibility checks on menopausal status (follicle-stimulating hormone levels $\geq 25 \mathrm{mIU} / \mathrm{mL}$ ), use of street drugs and alcohol (urine toxicology screen; blood ethanol), and general health status (comprehensive metabolic profile, thyroid stimulating hormone, HbAlc, complete blood count). Sixty-nine women were eligible for, and attended, the second research visit. At this visit, the Clinician Administered PTSD Scale for DSM-IV (CAPS) (Blake et al., 2000) was used to assess current (i.e., last 30 days) and past (i.e., worst lifetime episode) PTSD symptoms. Because the interview for past PTSD symptoms was added after data collection had begun, it was not administered to the first five particpants, and these participant are not included in the present study sample. Also, one woman did not meet the PTSD group definitions, described below, leaving 63 women for the current sample.

\section{Procedure}

Women participated in two research visits, beginning between 8 a.m. and 1 p.m., and were compensated $\$ 140.00$. Visit 1 began with three brief interviews to confirm intact cognitive functioning (Pfeiffer, 1975), absence of psychotic symptoms (First, Spitzer, Gibbon, \& Williams, 2001), and absence of active suicidal ideation (Kroenke \& Spitzer, 2002). This was followed by collection of the first sample of saliva and oral mucosal transudate (OMT). (Procedures for collecting oral fluids are described below, under Biological Measures). A nursing evaluation then assessed acute medical conditions, blood pressure, and body 
measurements. Blood was drawn via antecubital venipuncture and collected with appropriate anticoagulants for assessment of IL-6 and sIL-6R (sodium citrate), and the eligibility labs described above (EDTA for complete blood count and hemoglobin A1c, lithium heparin for all others). A urine sample was obtained for the toxicology screen and urinalysis. Women then completed demographic, health, and personality questionnaires, followed by collection of the second sample of saliva and OMT.

Visit 2 began with a brief interview to confirm absence of active suicidal ideation (Kroenke \& Spitzer, 2002). This was followed by collection of the first sample of saliva and OMT. A nursing evaluation then assessed acute medical conditions and blood pressure, and blood was drawn for assessment of IL-6 and sIL-6R as described above. Women then completed questionnaires and interviews about lifetime trauma, IPV, and PTSD symptoms (described below, under Psychosocial Measures). This was followed by collection of the second sample of saliva and OMT.

\section{Biological Measures}

Blood collection and isolation of plasma-Peripheral blood samples were kept refrigerated until delivered to the laboratory for processing. The tubes containing the blood were centrifuged for $10 \mathrm{~min}$ at $350 \mathrm{~g}$, the plasma separated by aspiration and aliquoted $(0.25$ $\mathrm{ml}$ ) into cryovials which were stored frozen at $-80^{\circ} \mathrm{C}$ until assay.

Collection of Saliva and OMT_-To collect saliva, participants used the Saliva Sampler (Saliva Diagnostic Systems, Brooklyn, NY). This device consists of a cellulose pad attached to a polypropylene stem. The pad is held under the tongue until a built-in indicator changes color, signaling collection of $1 \mathrm{ml}$ of saliva. The pad is then inserted into a tube containing 1 $\mathrm{ml}$ of preserving buffer solution. To collect OMT, participants used the OraSure collection device (OraSure Technologies, Bethlehem, PA), a cotton fiber pad treated with a hypertonic salt solution that enhances transport of OMT across the gingival crevice and oral mucosa (Ferri, 1998; Gallo, George, Fitchen, Goldstein, \& Hindahl, 1997). The pad is held between the lower cheek and gum for 3 minutes, and then inserted into a tube containing a preserving buffer solution. Following collection, oral fluids were kept at $4^{\circ} \mathrm{C}$ until processed, typically within the next 2 to 4 hours. To collect the samples for assay, the pads of both devices were separated from the rest of the applicator and pressed with the plunger of a $5 \mathrm{ml}$ syringe in order to release the fluid, which was collected in a $2 \mathrm{ml}$ microcentrifuge tube. After centrifugation $(60 \mathrm{~s}$ at $10,000 \mathrm{~g})$, the supernatants were transferred to a clean tube and kept frozen at $-80^{\circ} \mathrm{C}$ until assay.

Measurement of IL-6 and sIL-6R-The levels of IL-6 and sIL-6R were measured by commercial two-site ELISAs according to the manufacturer's instructions. An Opti-EIA kit (BD Pharmingen, San Diego, CA) was used for the measurement of IL-6, while a Duo Set R\&D kit (R\&D Diagnostics, Minneapolis, MN) was used for sIL-6R. Each sample was tested in triplicate; the average value was used for data analysis. Assay sensitivities were 0.3 $\mathrm{pg} / \mathrm{ml}$ and $1 \mathrm{pg} / \mathrm{ml}$ for IL-6 and sIL6R, respectively. Recovery of IL-6 from the Saliva and OMT collection devices in samples spiked with recombinant IL-6 (10 pg/ml) were 90 $\pm 9 \%$ and $93 \pm 5 \%$, respectively. Recovery of sIL-6R was $95 \pm 4 \%$ and $98 \pm 4 \%$ for samples spiked 
with recombinant sIL-6R $(1 \mathrm{ng} / \mathrm{ml})$ from the Saliva and OMT collection devices, respectively. Intra- and inter-assay coefficients of variation (CV) for the IL-6 and sIL-6R ELISAs were $6.9 \%$ and $9.6 \%$, and $8.6 \%$ and $6.4 \%$, respectively.

\section{Psychosocial Measures}

Emotional State-Ten items from the Positive and Negative Affect Scale (Watson, Clark, \& Tellegen, 1988) were used to assess the intensity of negative emotional state (e.g., distressed, upset; $1=$ very slightly or not at all, $5=$ extremely) at the beginning of each research visit, immediately following blood draws. Internal consistency coefficients were . 76 (visit 1) and .72 (visit 2).

Demographics and Health-Related Characteristics-Women reported their date of birth, current marital and employment status, ethnicity, highest level of education obtained, work schedule (i.e., day, second, night, or rotating shifts), annual household income, and whether they were regular smokers. Height and weight were used to calculate body mass index (BMI). At each visit, women reported (yes/no) whether they had smoked, drank caffeinated beverages, taken prescription or non-prescription medications (including vitamins or minerals), or exercised before arriving.

Because IL-6 and sIL-6R were assessed in oral fluids, in addition to plasma, three clinicallyvalidated items were included to assess participants' periodontal health. One item, "Do your gums usually bleed?," (yes/yes, when brushing my teeth/no) assessed self-reported symptoms of gingivitis. This item has sensitivity of .42 and specificity of .76, as indexed against a clinical evalution of bleeding on probe (Buhlin, Gustafsson, Andersson, Hakansson, \& Klinge, 2002). Two additional items, "Has any dentist/hygienist told you that you have deep pockets?" (Buhlin et al., 2002) (yes/no) and "Have you ever been told that you need periodontal or gum treatment?" (Pitiphat, Garcia, Douglass, \& Joshipura, 2002) (yes/no) have sensitvities of .55 and .65, and specificities of .90 and .64, respectively, as indexed against either number and depth of pockets, or radiographic determination of alveolar bone loss.

Trauma Load: Lifetime Trauma and Intimate Partner Violence-Trauma load (i.e., number of different types of traumas reported) predicts PTSD symptoms (Neuner et al., 2004) and their chronicity (Kolassa et al., 2010), and is therefore critical to interpreting group differences in the present study. The National Women's Study Event History Module (Resnick, 1996) assessed history (yes/no) of 10 different potentially traumatic events: serious accident; natural disaster; violent, unexpected loss of a family member; childhood sexual abuse; rape; sexual molestation; attempted sexual molestation; physical attack with a weapon; physical attack without a weapon but with intent to kill; and other extraordinarily stressful life event (events listed in this latter category were classified as potentially traumatic if they matched any of the other event cateories, or if the respondent reported fear of being seriously injured or killed). Also, to assess history of stalking by an intimate partner, women reported (yes/no) if (a) they had ever experienced any of eight events (e.g., unsolicited phone calls; unsolicited correspondence; vandalism; following or spying), and whether these events caused (b) fear, or (c) fear of bodily harm, threat of harm, or threat to 
one's own or another's life. Women meeting components a-c were classified as stalking victims (Tjaden \& Thoennes, 2000) Responses to this stalking assessment, and to the assessment of the ten potentially traumatic events, were summed to form a lifetime trauma load score, with a possible range of 0 to 11 .

To assess trauma load specific to intimate partner violence, the Revised Conflict Tactics Scale subscales-physical assault (12 items), sexual coercion (7 items), and psychological aggression (8 items) - were used (Straus, Hamby, \& Warren, 2003). For each item, women rated frequency of occurrence $(0=$ never, $6=$ more than 20 times $)$ with respect to their prior intimate relationships collectively. For the present purposes, items were scored dichotomously ( $0=$ never, $1=$ one or more times), and were summed to form an IPV-specific trauma load score, with a possible range of 0 to 27.

Depression Symptoms-The 10-item short form of the Center for Epidemiological Studies Depression Scale assessed frequency of depression symptoms in the past week $(0=$ rarely or none of the time - less than 1 day, $3=$ most or all of the time-5-7 days)

(Andresen, Malmgren, Carter, \& Patrick, 1994). This measure has high predictive accuracy when judged against the original 20-item scale, and high test-retest reliability. In the present sample, Cronbach's alpha was .77.

Posttraumatic Stress Disorder-The Clinician Administered PTSD Scale for DSM-IV (CAPS) (Blake et al., 2000) assessed current (i.e., last 30 days) and past (i.e., worst lifetime episode) PTSD symptoms. The CAPS interview was administered with respect to each woman's self-identified index event (i.e., most distressing potentially traumatic event), selected from lists of IPV events (e.g., hurt you physically, forced you into unwanted sexual activity) and non-IPV events (e.g., mugging, sexual victimization by anyone other than intimate partner, accident, natural disaster). For two women who reported none of the listed events, the most distressing event ever experienced (house burglarized; divorce aftermath) served as the index event.

Separate frequency and intensity ratings ( 0 to 4$)$ were made for each of the 17 PTSD symptoms. Twelve women reported that they had experienced no worst lifetime episode (i.e., no time period in which they were significantly more troubled by symptoms than during the past month). The majority of these women were almost entirely asymptomatic, with current PTSD symptom severity scores of $0(n=5)$, between 1 and $5(n=4)$, or between 12 and $27(n=3)$. Therefore, their past PTSD symptom scores were imputed from current scores.

Total symptom severity scores were computed as the sum of frequency and intensity ratings; internal consistency coefficients were .81 for current symptom severity, and .83 for past symptom severity. Full and partial/subthreshold diagnostic categories were computed for both current and past PTSD (Mylle \& Maes, 2004). Frequency and intensity ratings were translated into symptoms using the Rule of 3 (i.e., Freqency $>1 /$ Intensity $>2$, or Frequency $>$ 2/Intensity > 1) (Blanchard et al., 1995). A full PTSD diagnosis was considered present if the following criteria were met: B (at least one re-experiencing symptom), $\mathrm{C}$ (at least three avoidance symptoms), D (at least two hyperarousal symptoms), E (duration of episode at 
least 1 month), and $\mathrm{F}$ (at least a moderate level of distress, and/or functional impairment). Partial PTSD was defined as meeting diagnostic criteria for any two of three symptom clusters (B-D), along with criterion E; subthreshold PTSD was defined as having at least one symptom in each of the three symptom clusters (B-D), along with meeting criterion E. Because PTSD symptoms can arise after events that do not meet Criterion A (Anders, Frazier, \& Frankfurt, 2011; Mechanic, Weaver, \& Resick, 2008), this criterion was not required for diagnosis. In the present study, six of 37 women with past and/or current syndromal or subsyndromal PTSD symptoms did not fulfill Criterion A.

All interviews were audiotaped, and $20 \%$ were selected for reliability coding. Intraclass correlation coefficients for overall symptom severity were .93 (current symptoms) and .97 (past symtpoms). Kappa coefficients for diagnostic categories (no diagnosis, partial/ subthrehold diagnosis, or full diagnosis) were 1.00 (current) and .67 (past).

Using current and past PTSD diagnoses, three groups of women were defined. PTSD-/women $(n=26)$ met neither full nor partial/subthreshold diagnostic criteria for either current or past PTSD. PTSD+/- women $(n=22)$ met either full $(n=15)$ or partial/subthreshold $(n=$ 7) diagnostic criteria for past, but not current, PTSD. PTSD+/+ women $(n=15)$ met either full or partial/subthreshold diagnostic criteria for both past $\left(n_{\text {full }}=8, n_{\text {partial } / \text { subthreshold }}=7\right)$ and current $\left(n_{\text {full }}=2, n_{\text {partial/subthreshold }}=13\right.$ ) PTSD. One woman with a PTSD-/+ pattern was excluded from analysis.

\section{Data Analytic Strategy}

For categorical variables, group differences were evaluated by chi-square tests or Fisher's exact test when cells counts were low. For continuous variables, group differences were evaluated by ANOVAs for unbalanced designs or the non-parametric Kruskal-Wallis chisquare test for skewed variables. Tukey's studentized range test, alpha $=.05$, was used for post hoc tests; when following up Kruskal-Wallis tests, post hoc tests were conducted on ranks.

For IL-6 and sIL-6R measured in oral fluids, linear mixed effects models were fit to the data to test associations with three categorical variables: PTSD Group (PTSD-/-, PTSD+/-, PTSD+/+), and the repeated measures Visit (baseline/trauma assessment) and Sample (beginning of visit/end of visit). The fixed effects specification for each model included PTSD Group, Visit, Sample, all two-way interaction terms, and the three-way interaction term. The random effects specification for the models was random intercepts per participant. A direct product covariance structure for multivariate repeated measures was used, with an unstructured matrix for Visit and a compound symmetry structure for Sample (Moser, 2004). For IL-6 and sIL-6R measured in plasma, an identical analytic approach was used except that the model did not include a factor for Sample, and an unstructured covariance matrix was used. Mixed effects models were fit using SAS Proc Mixed (Moser, 2004), with Satterthwaite approximation for degrees of freedom. Hypothesis tests were conducted at the .05 significance level. Post hoc follow-up tests for statistically significant main effects and interactions were evaluated using Bonferroni-adjusted $p$-values. Because the distributions of IL-6 (measured in plasma and oral fluids) and sIL-6R (measured in oral fluids) were right-skewed, these variables were log-transformed, and results of statistical 
tests are summarized using geometric means and corresponding 95\% confidence intervals (Olivier, Johnson, \& Marshall, 2008).

\section{Results}

\section{Sample Characteristics}

As shown in Table 1, the three PTSD groups were quite similar to one another, with a few exceptions. For demographics, there were two statistically significant group differences. Among women employed outside the home $(n=53)$, those in the PTSD-/- group were more likely to work a standard day shift compared to the other two groups. Also, women in the PTSD+/+ group were about three years younger than those in the PTSD+/- group.

For health-related characteristics, there were no statistically significant group differences. In terms of stressor and symptom characteristics, as expected, women in the PTSD+/+ group reported the most severe current PTSD and depression symptoms; the scores of women in the other two groups did not differ from one another. Also as expected, women in the PTSD +/- and PTSD+/+ groups reported lifetime PTSD symptoms that were comparably severe, and more severe than those reported by women in the PTSD-/- group. Additionally, compared to women in the PTSD+/- group, women in the PTSD+/+ group reported that fewer years had passed since the index event. There were no statistically significant group differences in trauma load scores, although there was a trend for IPV-specific trauma load to be lower in the PTSD-/- group, compared to the other two groups. There were no statistically significant group differences in visit characteristics.

Time of sample collection was analyzed using a 3(PTSD Group) x 2(Visit) x 2(Sample) mixed-design ANOVA. A statistically significant effect for Sample, $F(1,59)=2497.90, p$ $<.0001$, was qualified by a Sample $\mathrm{x}$ Visit interaction, $F(1,59)=595.6, p<.0001$, which was in turn qualified by a Sample $\mathrm{x}$ Visit $\mathrm{x}$ PTSD Group interaction, $F(2,59)=3.77, p=$. 029. As expected, end-of-visit samples (13:09 p.m.) were collected later in the day than were beginning-of-visit samples (11:02 a.m.). Also, collection times for beginning-of-visit samples did not differ by visit (11:15 a.m. and 10:48 a.m. for baseline and trauma assessment visits, respectively, $p=.137$ ), whereas end-of-visit samples were collected later in the day at trauma assessment visits (12:33 p.m.) compared with baseline visits (11:15 a.m.) $(p<.0001)$. The three-way interaction revealed that, for each PTSD Group, there were no statistically significant visit differences in collection times for beginning-of-visit samples ( $p$ s .103 to .854). In contrast, end-of-visit samples were collected later in the day at the trauma assessment visit compared with the baseline visit for the PTSD-/- $(p=.003)$ and PTSD+/+ groups $(p=.023)$, but not for the PTSD+/- group $(p=.121)$ (see Table 1$)$.

\section{Emotional State}

Ratings of negative emotional state made at the start of each visit were right-skewed, and transformations did not normalize the distributions. However, these ratings were analyzed with a 3(PTSD Group) x 2(Visit) mixed-design ANOVA because there is no straightforward nonparametric analogue for this test. Results yielded a statistically significant effect of PTSD Group, $F(2,60)=6.33, p=.003$. There were no other statistically significant effects 
( $p s>.770)$. Post hoc tests showed that, at the beginning of the research visits, the PTSD $+/+$ group reported a more intense negative emotional state $(M=13.43, S D=2.77)$ than did the PTSD+/- group ( $M=11.25, S D=1.13)$ and the PTSD-/- group $(M=11.42, S D=2.04)$; the latter two groups did not differ from one another. Two nonparametric Kruskal-Wallis tests that evaluated PTSD Group effects separately by visit supported the same conclusion.

\section{IL-6 and sIL-6R: The Roles of PTSD Group and Psychological Context}

Mixed effects models were used to test the hypothesized relationships separately for IL-6 and sIL-6R levels measured in each of the three biological fluids—saliva, OMT, and plasma. Based on the results of these models, geometric means and corresponding $95 \%$ confidence intervals are presented for IL-6 (Figures 1a-b) and sIL-6R (Figures 2a-b) by PTSD group, sample (beginning and end of visit—for oral fluids only), and visit (baseline and trauma assessment).

IL-6 Levels-The first question that was addressed concerned whether IL-6 levels differed by PTSD diagnostic status, and specifically whether PTSD+/- women showed lower IL-6 levels than PTSD+/+ women. This was tested by evaluating by the main effect of PTSD Group for each of the three mixed models. There were no statistically significant main effects for PTSD Group for IL-6 measured in saliva $(p=.807)$, OMT $(p=.748)$, or plasma $(p=.254)$.

The second question that was addressed concerned whether IL-6 levels were greater at the beginning of the visits, suggesting an anticipatory response, or at the end of the visits, suggesting a reactivity response. Also of interest was whether these responses were greater for PTSD+/+ women compared to women in the other two groups, and at the second visit compared to the first, which would suggest an elevated IL- 6 response by PTSD+/+ women to trauma-related cues (i.e., the trauma assessment and diagnostic interview). Main effects for Sample, and the interaction terms for PTSD Group x Sample, and PTSD Group x Sample $x$ Visit were evaluated, respectively, to test these associations. These effects were tested for IL-6 measured in saliva and OMT, not plasma, because only oral fluids were collected at both the beginning and end of visits.

For salivary IL-6, a statistically significant main effect of Sample revealed higher levels at the beginning of the visits $(M=1.74 \mathrm{pg} / \mathrm{ml}, 95 \%$ CIs $[1.32,2.34])$ than at the end $(M=1.51$ $\mathrm{pg} / \mathrm{ml}, 95 \%$ CIs $[1.12,2.04]), F(1,61.5)=8.46, p=.005$. For IL-6 measured in OMT, there was no statistically significant main effect of Sample $(p=.317)$.

The PTSD Group x Sample interaction term approached statistical significance for salivary IL-6, $F(2,61.6)=2.94, p=.060$, and reached statistical significance for IL-6 measured in OMT, $F(2,66.9)=3.94, p=.024$. To examine these interactions, two sets of follow-up tests were conducted. The first set examined whether there were differences in IL-6 levels among the three PTSD groups either at the beginning of the visits, or at the end of the visits; these tests yielded no statistically significant group differences for IL-6 measured in saliva ( $p \mathrm{~s}=$. 642 to .763 ) or in OMT ( $p s=.531$ to .788). The second set of follow-up tests examined, for each PTSD group, whether there were differences in IL-6 levels at the beginning verus the end of the visits. For salivary IL-6, the PTSD+/+ women showed higher levels at the 
beginning of the visits $(M=2.09 \mathrm{pg} / \mathrm{ml}, 95 \%$ CIs $[1.15,3.89])$ compared to the end, $(M=$ $1.41 \mathrm{pg} / \mathrm{ml}, 95 \%$ CIs $[0.71,2.75]) t(60.7)=3.34, p=.022$. In contrast, there were no statistically significant differences between beginning and end of visit salivary IL-6 levels for PTSD-l- women $(t=0.86, p=.395)$ or PTSD $+/-$ women $(t=0.50, p=.618)$. For IL-6 measured in OMT, there was an identical pattern (i.e., levels at the beginning of the visits exceeded those at the end, but only for PTSD+/+ women), however this effect did not survive the Bonferroni correction $(p=.239)$.

The PTSD Group x Sample $x$ Visit interaction term was not statistically significant for IL-6 measured in saliva $(p=.546)$ or OMT $(p s=.357)$. Two other effects approached statistical significance, but follow-up tests did not survive Bonferroni correction. Specifically, for salivary and plasma IL-6 levels, there were trends for a PTSD Group x Visit interaction, $F_{\text {saliva }}(2,58.3)=2.96, p=.059, F_{\text {plasma }}(2,60)=2.81, p=.068$. These reflected tendencies for the PTSD-/- group to show higher IL-6 levels at the first visit compared to the second (salivary and plasma IL-6) and, at the first visit, to show IL-6 levels that were higher than those of the PTSD+/- group (plasma IL-6). There were no other statistically significant effects for IL-6 measured in saliva ( $p s=.822$ to .883 ), OMT ( $p s=.395$ to .928 ), or plasma $(p=.906)$.

sIL-6R Levels-The question of whether sIL-6R levels differed by PTSD diagnostic. status was tested by evaluating the main effect of PTSD Group for each of the three mixed models - saliva, OMT, and plasma. For plasma sIL-6R levels there was a statistically significant effect of PTSD Group, $F(2,59.9)=3.07, p=.054$. Plasma sIL-6R levels were higher in the PTSD+/+ group $(32.98 \pm 2.16 \mathrm{ng} / \mathrm{ml})$ compared to the PTSD+/- group $(26.10 \pm 1.78 \mathrm{ng} / \mathrm{ml}), t(59.7)=-2.46, p=.05$. Levels in the PTSD- - group $(28.27 \pm 1.64$ $\mathrm{ng} / \mathrm{ml}$ ) did not differ from those in the other two groups. There were no statistically significant PTSD Group effects for sIL-6R measured in saliva $(p=.876)$ or OMT $(p=.951)$.

Sample main effects were evaluated to test whether sIL-6R levels were greater at the beginning of the visits, suggesting an anticipatory response, or at the end of the visits, suggesting a reactivity response. There was a statistically significant Sample main effect for sIL-6R measured in saliva, $F(1,60.3)=10.09, p=.002$, and in OMT, $F(1,59.6)=10.57, p$ $=.002$. These effects revealed that sIL-6R levels in both oral fluids were greater at the end of the visits (saliva: $M=54.95 \mathrm{ng} / \mathrm{ml}, 95 \%$ CIs [42.66, 69.18]; OMT: $M=83.18 \mathrm{ng} / \mathrm{ml}, 95 \%$ CIs [67.61, 102.33]) than at the beginning (saliva: $M=41.69 \mathrm{ng} / \mathrm{ml}, 95 \%$ CIs[32.36, 53.70]; OMT: $M=72.44 \mathrm{ng} / \mathrm{ml}, 95 \%$ CIs $[58.88,89.13])$.

The PTSD Group x Sample interaction term did not reach statistical significance for either saliva $(p=.645)$ or OMT $(p=.719)$. Similarly, the PTSD Group $\mathrm{x}$ Sample $\mathrm{x}$ Visit interaction term did not reach statistical significance for either saliva $(p=.438)$ or OMT ( $p$ $=.812)$. There were no other statistically significant effects for sIL-6R measured in saliva ( $p$ s from .411 to .843), OMT ( $p$ s from .242 to .749), or plasma ( $p$ s from .611 to .923).

Emotional State, Salivary IL-6, and Plasma sIL-6R-To further examine the idea that connections between PTSD group and salivary IL-6 are sensitive to psychological context, as reflected in the PTSD Group x Sample interaction reported above, correlations 
between intensity of negative emotions and salivary IL-6 levels measured at the beginning of visits were computed by PTSD group. All values were averaged across visits, given that there were no visit effects. Focus is on the magnitude of correlations, rather than statistical significance, given the small sample size for each group. Spearman's rank order correlations between intensity of negative emotional state and levels of salivary IL- 6 were $-.02,-.35$, and +.46 for the PTSD $-/-,+/-$, and $+/+$ groups, respectively. We explored these correlations for plasma sIL-6R levels, which differed by PTSD group, but were measured only at the beginning of the visits. Rank order correlations between negative emotional state and plasma sIL-6R levels were $+.003,-.15$, and +.47 , for the PTSD-/-, +/- and $+/+$ groups, respectively.

\section{Follow-up Analyses}

Mixed effects models for salivary IL-6 and plasma sIL-6R levels were re-run adjusting for years since index event, shift status (coded day shift/not employed outside the home vs. other than day shift), or sample collection time (included as a time-varying covariate and coded in 24-hour clock time) given that these variables were associated with PTSD group. Neither years since index event nor shift status was a statistically significant predictor ( $p \mathrm{~s}=$. 341 to .911). After adjusting for each variable, all effects involving PTSD Group reached an identical or more stringent level of statistical significance, except in the plasma sIL-6R model adjusted for shift work ( $p=.058$ versus .054 without adjustment). When sample collection time was included as a time-varying covariate, the PTSD Group main effect for plasma sIL-6R was reduced (from $p=.054$ to $p=.069$ ), although the effect for sample collection time was not statistically significant $(p=.175)$. For salivary IL-6, the PTSD Group x Sample interaction retained trend status, changing from $p=.060$ to $p=.058$ ), with no statistically significant effect for sample collection time $(p=.509)$.

Two other variables known to influence inflammatory mediators-BMI and age-were not included in the mixed models a priori because of the low participant to variable ratio, particularly for the oral fluid models, and the potential for overfitting the models (Babyak, 2004). BMI was not associated with PTSD group, and age was associated in a direction that would not be confounding (i.e., PTSD+/+ women were younger than those in the PTSD+/group), yet these two variables could still potentially affect the biological measures.

Therefore, the mixed effects models for salivary IL- 6 and plasma sIL-6R levels were re-run adjusting for BMI and age simultaneously. Neither BMI nor age was a statistically significant predictor ( $p s=.257$ to .890 , respectively). After adjusting for both variables, all effects involving PTSD group reached an identical or more stringent level of statistical significance.

The PTSD+/+ group, compared to the other two groups, showed more severe current symptoms of depression. This makes it tempting to ask whether the observed patterns for salivary IL-6 and plasma sIL-6R are "due to" comorbid depression (Gill et al., 2010; Maes et al., 1999). Without diagnostic interviews for depression, and without sufficient participants to form PTSD+/+ groups with and without comorbid depression, this question is difficult to assess in the present design. Indeed, it is well-recognized that depression and PTSD share core symptoms (Tsai, Pietrzak, Southwick, \& Harpaz-Rotem, 2011), and that 
statistically adjusting for depression symptoms in a situation such as the present one will not "equalize" the groups on this dimension and may remove variance that rightly belongs to the construct of PTSD (Miller \& Chapman, 2001). Keeping these limitations in mind, mixed effects models were re-run to test whether depression symptoms predicted salivary IL-6 and plasma sIL-6R levels, and whether PTSD group contributed beyond symptoms of depression. For salivary IL-6 levels, there were no statistically significant associations with depression symptoms, and with depression symptoms in the model, the PTSD Group $\mathrm{x}$ Sample interaction retained is original level of significance $(p=.060)$. For plasma sIL-6R levels, there were no statistically significant associations with depression symptoms, yet with depression symptoms in the model, the PTSD Group term no longer reached statistical significance ( $p=.103$ ). This implies that elevations in plasma sIL-6R are predicted by the shared, rather than unique, aspects of depression and PTSD.

\section{Discussion}

Understanding connections between the cytokine IL-6 and PTSD is of interest in terms of explaining why this stress-related emotional disorder predicts poorer physical health (Ahmadi et al., 2011). The present study examined how classifying participants on both past and current symptom severity affects connections with IL-6, and tested whether these connections are dynamic, or sensitive to psychological context. Results provide partial support for these ideas, and underscore the need for more study of sIL-6R.

The first question addressed here concerned whether IL-6 levels would be lower among women who reported lifetime decreases in PTSD symptom severity (PTSD+/-) compared to those who did not (PTSD+/+). This was spurred by our prior report showing that severe past PTSD symptoms predicted lower IL-6 levels (Newton et al., 2013), and the question of whether this held only for persons whose past symptoms subsided. This hypothesis was not supported. The present results revealed no statistically significant PTSD group main effect for IL-6 levels measured in saliva, plasma, or OMT. Nonetheless, it may be premature to abandon this question. Although the differences were not statistically significant, review of Figure 1 shows a general pattern of lower IL-6 levels in plasma and perhaps saliva (but not OMT) for women in the PTSD+/- group. Further, PTSD+/+ women had relatively low symptom levels, with only two reaching syndromal levels. Thus, the current patterns may underestimate group differences that will emerge when persons with fully syndromal PTSD are studied. Indeed, Gill et al. (2013) recently reported that women recovered from PTSD showed plasma IL-6 levels significantly lower than those of women with persistent PTSD, and comparable to those of never-traumatized controls. Overall, then, further evaluation of this question seems warranted.

The second question addressed here concerned whether connections between IL- 6 and lifetime PTSD status are dynamic, or sensitive to psychological context, and, if so, whether this sensitivity is characterized by anticipatory processes or by reactivity to trauma-related cues. Tentative support for the importance of anticipatory processes was observed. Women in the PTSD+/+ group, compared with the other two groups, were characterized by apparent sensitivity to anticipatory states, as reflected by a more intense negative emotional state at the beginning of the visits, higher salivary IL-6 levels at the beginning compared with the 
end of the visits, and positive correlations between negative emotional state and both salivary IL-6 and sIL-6R, all measured at the beginning of the visits. These effects were not moderated by visit type, suggesting they may be general responses to anticipatory states, rather than to anticipation of trauma-related cues per se. However, the possibility that the PTSD+/+ group perceived both visits to be trauma-related cannot be ruled out. In addition, because emotional state was not measured at the end of the visits, we cannot rule out the possibility that effects for this measure are due to trait, rather than state, differences between PTSD groups. Because of these limitations, our interpretation should be considered tentative. Nevertheless, the overall pattern does suggest the hypothesis that anticipatory states, and aversive anticipation in particular, may be important for the regulation of inflammation in PTSD.

Interestingly, anticipatory states feature prominently in neural models of anxiety disorders, and in models of stress and health. Compared to non-anticipatory states or tasks (Chua, Krams, Toni, Passingham, \& Dolan, 1999; Simmons, Matthews, Stein, \& Paulus, 2004), and compared to anticipation of positive stimuli (Simmons et al., 2011), anticipation of aversive or emotionally negative stimuli reliably recruits a common neural circuitry (i.e., the insular cortex, anterior cingulate cortex, and areas of the prefrontal cortex). Further, this circuitry's connection with potentially modulating brain regions (e.g., the amygdala and areas of the prefrontal cortex) differs for persons with and without anxiety disorders, including PTSD (Aupperle et al., 2012; Simmons et al., 2008). It is tempting to speculate that the response profile observed in the PTSD+/+ group may be a peripheral signature of anxiety-linked neural circuitry recruited during anticipation. Regarding health, anticipatory responses are considered part of "perseverative cognition," or stress-related mental activity that occurs before and after the stressor per se, and that prolongs physiological stress arousal (Brosschot, Gerin, \& Thayer, 2006, p. 113). If proinflammatory cytokines are activated by anticipatory stress among persons with PTSD, it seems reasonable to hypothesize that repeated episodes of anticipation may increase overall lifetime exposure to these cytokines, and to other proinflammatory proteins, thereby influencing health.

The absence of a reactivity pattern (i.e., higher IL-6 at the end of the research visit compared to the beginning) among women in the PTSD+/+ group might seem unexpected, especially at the trauma-related assessment. After all, physiological reactivity to trauma cues is widely documented (Peirce, Newton, Buckley, \& Keane, 2002). However, while a diagnostic interview does include exposure to trauma reminders, this occurs in a supportive, empathic interaction that contains and limits distress. Another research group reported that, in persons with PTSD, salivary cortisol decreased over the course of a diagnostic interview (Kolassa et al., 2007). On the other hand, another study of persons with PTSD reported either decreases or no changes in an array of chemokines, acute-phase proteins, and cytokines measured both before and after exposure to individualized trauma scripts that were designed to provoke reactivity (Nowotny et al., 2010). Thus, perhaps for cytokines and related proteins, exposure to trauma cues does not generate a classic reactivity pattern.

The IL-6 patterns described above do have other potential explanations that will need to be evaluated. For example, daily variability in IL-6 levels (Picotte, Campbell, \& Thorland, 2009), or unmeasured health behaviors (O'Connor et al., 2009), could contribute to these 
patterns, as could altered circadian IL-6 rhythms (Alesci et al., 2005). Also, statistically significant effects were apparent only for IL-6 measured in saliva. Salivary IL-6 may have the greatest sensitivity to the psychological factors examined here. Studies have shown, at best, small correlations between IL-6 measured in saliva and serum (Sjogren et al., 2006) and in saliva and plasma (Fernandez-Botran et al., 2011), and in our prior research we observed only moderate correlations between IL-6 measured in saliva and OMT (FernandezBotran et al., 2011). Being produced by salivary gland cells, salivary IL-6 may be subject to unique, local regulation (Sjogren et al., 2006; Tanda et al., 1998). Collecting multiple fluids, therefore, may help trace the sources of psychological influences on inflammatory mediators.

In the third and final aim of this study, questions about PTSD group and psychological context were extended to sIL-6R. Women in the PTSD+/+ group showed higher plasma sIL-6R levels than those in the other two groups; levels in the PTSD+/- and PTSD-/groups did not differ from one another. This adds to the small literature documenting connections between trauma-related psychological distress (Maes et al., 1999; Miller et al., 2001) — especially persistent distress (Sutherland et al., 2003)—and sIL-6R elevations. Because soluble cytokine receptors act as endogenous regulators of cytokine activity in vivo, they are essential to understanding cytokine signaling and its consequences (Heaney \& Golde, 1996). In the case of the soluble form of the IL-6 receptor, it acts as an agonist, amplifying and prolonging the actions of IL-6, and broadening the range of target cells and tissues (Rose-John et al., 2006). It has also been argued that sIL-6R plays a critical role in the immunological processes that contribute to the "transition from acute to chronic inflammation" (Rose-John et al., 2006, p. 227), and in mediating the proinflammatory activities of IL-6 (Scheller et al., 2011). Thus, sIL-6R may be key to evaluating the plausibility of the proinflammatory model of PTSD, at least within the context of IL-6 (Rose-John et al., 2006; Scheller et al., 2011). Because blood was drawn only at the beginning of the visits, our design does not enable us to determine if connections between sIL-6R and PTSD are limited to anticipatory states. Also, compared to sIL-6R levels in plasma, those measured in oral fluids exhibited a different pattern of correlations: They did not vary by PTSD group, but were significantly greater at the end of the research visits, compared to the beginning. We are not aware of published data examining sIL-6R in the context of acute stressors, or testing whether it responds to acute stressors with a different time course compared to IL-6. Overall, whether measured in plasma or oral fluids, the regulation of sIL-6R differed from that of IL-6.

The present study has a number of limitations. First, although we used standard diagnostic procedures, lifetime PTSD symptoms were assessed retrospectively and are therefore subject to the fallibility of memory. They were imputed for some women, but most of these women were currently asymptomatic and stated that they had never experienced a more severe symptom episode. Also, the CAPS was administered to assess symptoms related to IPV, or to the worst lifetime trauma, rather than to multiple traumas, a procedure that may have missed symptoms and influenced group classification. Second, the patterns observed here should not be generalized to men, to premenopausal women, or to persons who have more recently experienced a traumatic event. Third, the results for plasma IL-6 levels diverge somewhat from the pattern of results in our prior study, where we used continuous measures 
of past and current PTSD symptom severity, and treated them as independent predictors (Newton et al., 2013). In this approach, as mentioned above, more severe past symptoms predicted lower plasma IL-6 levels at the baseline visit, whereas the present study observed no statistically significant group differences in plasma IL-6 levels. In addition, in our prior study but not here, women with severe past PTSD symptoms showed greater plasma IL-6 levels at the beginning of the second visit versus the first, suggesting a response that was specific to anticipating trauma reminders. We attribute these differences between studies to the fact that the two analytic approaches have different statistical power, and ask somewhat different questions of the data. The use of categorical groups, compared to continuous measures, decreases statistical power and therefore requires a larger sample size to detect effects. Thus, for example, past PTSD symptom severity may indeed predict stronger IL-6 elevations while anticipating trauma reminders, but this may be harder to detect when severe past symptoms are distributed across two different groups-remitted and persistent. Finally, although we assessed oral health using self-report measures validated against clinical indicators, the sensitivity of these instruments may have caused lack of identification of some persons with oral health problems, potentially influencing the oral fluid biological measures.

By differentiating lifetime PTSD status rather than measuring current symptoms alone, the present study raises questions about connections between recovery from emotional trauma and the regulation of inflammatory mediators (Gill, Saligan, Lee, Rotolo, \& Szanton, 2013). Specifically, the three PTSD groups described here may reflect different symptom trajectory patterns that have been identified in studies of trauma recovery: relative resistance to PTSD $(-/-)$, remission or recovery from PTSD (+/-), and persistent PTSD (+/+) (Berntsen et al., 2012; Bonanno et al., 2006; Hobfoll et al., 2009; Layne et al., 2007). From this perspective, the present data suggest that regulation of inflammatory mediators is connected with the ability to overcome clinically significant stress reactions: among women recovered from PTSD, compared to those with persistent symptoms, salivary IL-6 levels were not sensitive to anticipatory states, plasma sIL-6R levels were lower, and there were hints that plasma and perhaps salivary IL-6 levels were regulated within a narrow range. It is also possible, however, that the IL-6 and sIL-6R patterns observed here are state markers-patterns that change as symptoms wax and wane-or, for the PTSD+/+ group, that will subside with further recovery. Indeed, the PTSD+/+ group was 10 fewer years removed from their index trauma compared to the PTSD+/- group. Yet, it is notable that time since index trauma predicted neither salivary IL-6 nor plasma sIL-6R levels. Longitudinal and experimental study designs will be helpful to evaluate these possibilities.

\section{Acknowledgments}

Related reports from this database have been published (Fernandez-Botran, Miller, Burns, \& Newton, 2011; Newton et al., 2013; Newton et al., 2011). This research was supported by funding from the National Institute on Aging, and by a Research on Women grant from the University of Louisville. We would like to acknowledge the support of the University of Louisville Hospital Clinical Research Center. 


\section{References}

Ahmadi N, Hajsadeghi F, Mirshkarlo HB, Budoff M, Yehuda R, Ebrahimi R. Post-traumatic stress disorder, coronary atherosclerosis, and mortality. The American Journal of Cardiology. 2011; 108(1):29-33.10.1016/j.amjcard.2011.02.340 [PubMed: 21530936]

Al'Absi M, Lovallo WR. Cortisol concentrations in serum of borderline hypertensive men exposed to a novel experimental setting. Psychoneuroendocrinology. 1993; 18(5/6):355-363. [PubMed: 8416046]

Alesci S, Martinez PE, Kelkar S, Ilias I, Ronsaville DS, Listwak SJ, Gold PW. Major depression is associated with significant diurnal elevations in plasma interleukin-6 levels, a shift of its circadian rhythm, and loss of physiological complexity in its secretion: Clinical implications. Journal of Clinical Endocrinology \& Metabolism. 2005; 90(5):2522-2530.10.1210/jc.2004-1667 [PubMed: 15705924]

Anders SL, Frazier PA, Frankfurt SB. Variations in Criterion A and PTSD rates in a community sample of women. Journal of Anxiety Disorders. 2011; 25(2):176-184.10.1016/j.janxdis. 2010.08.018 [PubMed: 20888184]

Andresen EM, Malmgren JA, Carter WB, Patrick DL. Screening for depression in well older adults: Evaluation of a short form of the CES-D. American Journal of Preventive Medicine. 1994; 10(2): 77-84. [PubMed: 8037935]

Aupperle RL, Allard CB, Grimes EM, Simmons AN, Flagan T, Behrooznia M, Stein MB. Dorsolateral prefrontal cortex activation during emotional anticipation and neuropsychological performance in posttraumatic stress disorder. Archives of General Psychiatry. 2012; 69(4):360-371.10.1001/ archgenpsychiatry.2011.1539 [PubMed: 22474105]

Babyak MA. What you see may not be what you get: A brief, nontechnical introduction to overfitting in regression-type models. Psychosomatic Medicine. 2004; 66:411-421.10.1097/01.psy. 0000127692.23278.a9 [PubMed: 15184705]

Berntsen D, Johannessen KB, Thomsen YD, Bertelsen M, Hoyle RH, Rubin DC. Peace and war: trajectories of posttraumatic stress disorder symptoms before, during, and after military deployment in afghanistan. Psychological Science. 2012; 23(12):1557-1565.10.1177/0956797612457389 [PubMed: 23129059]

Blake, D.; Weathers, F.; Nagy, L.; Kaloupek, D.; Klauminzer, D.; Charney, D.; Buckley, TC. Instruction Manual: Clinician Administered PTSD Scale (CAPS). Boston, MA and West Haven, CT: Behavioral Sciences Division and Neurosciences Division, National Center for Posttraumatic Stress Disorder; 2000.

Blanchard EB, Hickling EJ, Taylor AE, Forneris CA, Loos W, Jaccard J. Effects of varying scoring rules of the Clinician-Administered PTSD Scale (CAPS) for the diagnosis of post-traumatic stress disorder in motor vehicle accident victims. Behaviour Research and Therapy. 1995; 33(4):471475. [PubMed: 7755537]

Bonanno GA, Galea S, Bucciarelli A, Vlahov D. Psychological resilience after disaster - New York City in the aftermath of the September 11th terrorist attack. Psychological Science. 2006; 17(3): 181-186.10.1111/j.1467-9280.2006.01682.x [PubMed: 16507055]

Brosschot JF, Gerin W, Thayer JF. The perseverative cognition hypothesis: A review of worry, prolonged stress-related physiological activation, and health. Journal of Psychosomatic Research. 2006; 60(2):113-124.10.1016/j.jpsychores.2005.06.074 [PubMed: 16439263]

Buhlin K, Gustafsson A, Andersson K, Hakansson J, Klinge B. Validity and limitations of selfreported periodontal health. Community Dentistry and Oral Epidemiology. 2002; 30:431-437. [PubMed: 12453114]

Chiang JJ, Eisenberger NI, Seeman TE, Taylor SE. Negative and competitive social interactions are related to heightened proinflammatory cytokine activity. Proceedings of the National Academy of Sciences. 2012; 109(6):1878-1882.10.1073/pnas.1120972109

Chua P, Krams M, Toni I, Passingham R, Dolan R. A functional anatomy of anticipatory anxiety. Neuroimage. 1999; 9(6):563-571.10.1006/nimg.1999.0407 [PubMed: 10334900]

Dawson DA, Grant BF, Stinson FS, Zhou Y. Effectiveness of the derived Alcohol Use Disorders Identification Test (AUDIT-C) in screening for alcohol use disorders and risk drinking in the US 
general population. Alcoholism: Clinical and Experimental Research. 2005; 29(5):844854.10.1097/01.alc.0000164374.32229.a2

Fernandez-Botran R, Miller JJ, Burns VE, Newton TL. Correlations among inflammatory markers in plasma, saliva and oral mucosal transudate in post-menopausal women with past intimate partner violence. Brain, Behavior, and Immunity. 2011; 25(2):314-321.10.1016/j.bbi.2010.09.023

Ferri RS. Oral mucosal transudate testing for HIV-1 antibodies: A clinical update. Journal of the Association of Nurses in AIDS Care. 1998; 9(2):68-72.10.1016/s1055-3290(98)80062-9 [PubMed: 9513137]

First, MB.; Spitzer, RL.; Gibbon, M.; Williams, JBW. Structured Clinical Interview for DSM-IV-TR Axis I Disorders, Research Version, Patient Edition (SCID I/P). New York: New York State Psychiatric Institute. Biometrics Research Department; 2001. (2/2001 revision ed.)

Gabay C, Kushner I. Acute-phase proteins and other systemic responses to inflammation. New England Journal of Medicine. 1999; 340(6):448-454.10.1056/NEJM199902113400607 [PubMed: 9971870]

Gallo D, George JR, Fitchen JH, Goldstein AS, Hindahl MS. Evaluation of a system using oral mucosal transudate for HIV-1 antibody screening and confirmatory testing. Journal of the American Medical Association. 1997; 277(3):254-258.10.1001/jama.277.3.254 [PubMed: 9005276]

Gill J, Luckenbaugh D, Charney D, Vythilingam M. Sustained elevation of serum interleukin-6 and relative insensitivity to hydrocortisone differentiates posttraumatic stress disorder with and without depression. Biological Psychiatry. 2010; 68(11):999-1006.10.1016/j.biopsych.2010.07.033 [PubMed: 20951370]

Gill JM, Saligan L, Lee H, Rotolo S, Szanton S. Women in recovery from PTSD have similar inflammation and quality of life as non-traumatized controls. Journal of Psychosomatic Research. 2013; 74(4):301-306.10.1016/j.jpsychores.2012.10.013 [PubMed: 23497831]

Gill JM, Saligan L, Woods S, Page G. PTSD is associated with an excess of inflammatory immune activities. Perspectives in Psychiatric Care. 2009; 45(4):262-277. [PubMed: 19780999]

Heaney M, Golde D. Soluble cytokine receptors. Blood. 1996; 87(3):847-857. [PubMed: 8562952]

Hobfoll SE, Palmieri PA, Johnson RJ, Canetti-Nisim D, Hall BJ, Galea S. Trajectories of resilience, resistance, and distress during ongoing terrorism: The case of Jews and Arabs in Israel. Journal of Consulting and Clinical Psychology. 2009; 77(1):138-148.10.1037/a0014360 [PubMed: 19170460]

Kolassa I-T, Eckart C, Ruf M, Neuner F, de Quervain DJF, Elbert T. Lack of cortisol response in patients with posttraumatic stress disorder (PTSD) undergoing a diagnostic interview. BMC Psychiatry. 2007; 7(54)10.1186/1471-244X-7-54

Kolassa IT, Ertl V, Eckart C, Kolassa S, Onyut LP, Thomas E. Spontaneous remission from PTSD depends on the number of traumatic event types experienced. Psychological Trauma: Theory, Research, Practice, and Policy. 2010; 2(3):169-174.10.1037/a0019362

Kroenke K, Spitzer RL. The PHQ-9: A new depression diagnostic and severity measure. Psychiatric Annals. 2002; 32(9):1-7.

Layne, CM.; Warren, JS.; Watson, PJ.; Shalev, AY. Risk, vulnerability, resistance, and resilience: Toward an integrative conceptualization of posttraumatic adaptation. In: Friedman, MJ.; Keane, TM.; Resick, PA., editors. Handbook of PTSD: Science and Practice. New York: Guilford; 2007. p. 497-520.

Maes M, Lin A-h, Delmeire L, Van Gastel A, Kenis G, De Jongh R, Bosmans E. Elevated serum interleukin-6 (IL-6) and IL-6 receptor concentrations in posttraumatic stress disorder following accidental man-made traumatic events. Biological Psychiatry. 1999; 45(7):833-839.10.1016/ s0006-3223(98)00131-0 [PubMed: 10202570]

McCanlies EC, Araia SK, Joseph PN, Mnatsakanova A, Andrew ME, Burchfiel CM, Violanti JM. Creactive protein, interleukin-6, and posttraumatic stress disorder symptomology in urban police officers. Cytokine. 2011; 55(1):74-78.10.1016/j.cyto.2011.03.025 [PubMed: 21493089]

Mechanic MB, Weaver TL, Resick PA. Mental health consequences of intimate partner abuse: A multidimensional assessment of four different forms of abuse. Violence Against Women. 2008; 14(6):634-654.10.1177/1077801208319283 [PubMed: 18535306] 
Miller GA, Chapman JP. Misunderstanding analysis of covariance. Journal of Abnormal Psychology. 2001; 110(1):40-48.10.1037//0021-843x.110.1.40 [PubMed: 11261398]

Miller RJ, Sutherland AG, Hutchison JD, Alexander DA. C-reactive protein and interleukin 6 receptor in post-traumatic stress disorder: a pilot study. Cytokine. 2001; 13(4):253-255.10.1006/cyto. 2000.0825 [PubMed: 11237435]

Moser EB. Repeated Measures Modeling with Proc Mixed. SAS Users Group International Conference. 2004; 188-29:1-19. http://www2.sas.com/proceedings/sugi29/toc.html\#stat.

Mylle J, Maes M. Partial posttraumatic stress disorder revisited. Journal of Affective Disorders. 2004; 78(1):37-48.10.1016/s0165-0327(02)00218-5 [PubMed: 14672795]

Neuner F, Schauer M, Karunakara U, Klaschik C, Robert C, Elbert T. Psychological trauma and evidence for enhanced vulnerability for posttraumatic stress disorder through previous trauma among West Nile refugees. Bmc Psychiatry. 2004; 4:34.10.1186/1471-244X-4-34 [PubMed: 15504233]

Newton TL, Fernandez-Botran R, Miller JJ, Cambon AC, Burns VE, Allison KE. Posttraumatic stress symptom severity and inflammatory processes in midlife women. Psychological Trauma: Theory, Research, Practice, and Policy. 2013; 5(5):439-447.

Newton TL, Fernandez-Botran R, Miller JJ, Lorenz DJ, Burns VE, Fleming KN. Markers of inflammation in midlife women with intimate partner violence histories. Journal of Women's Health. 2011; 20(12):1871-1880.10.1089/jwh.2011.2788

Nishanian P, Azia N, Chung J, Detels R, Fahey JL. Oral fluids as an alternative to serum for measurement of markers of immune activation. Clinical and Diagnostic Laboratory Immunology. 1998; 5(4):507-512. [PubMed: 9665958]

Nowotny B, Cavka M, Herder C, Löffler H, Poschen U, Joksimovic L, Kruse J. Effects of acute psychological stress on glucose metabolism and subclinical inflammation in patients with posttraumatic stress disorder. Hormone and Metabolic Research. 2010; 42:746-753.10.1055/ s-0030-1261924 [PubMed: 20665427]

O'Connor MF, Bower JE, Cho HJ, Creswell JD, Dimitrov S, Hamby ME, Irwin MR. To assess, to control, to exclude: Effects of biobehavioral factors on circulating inflammatory markers. Brain, Behavior, and Immunity. 2009; 23(7):887-897.10.1016/j.bbi.2009.04.005

Ohman, A.; Hamm, A.; Hugdahl, K. Cognition and the autonomic nervous system: Orienting, anticipation, and conditioning. In: Cacioppo, JT.; Tassinary, LG.; Bernston, GG., editors. Handbook of Psychophysiology. 2. New York NY: Cambridge University Press; 2000. p. 533-575.

Olivier J, Johnson WD, Marshall GD. The logarithmic transformation and the geometric mean in reporting experimental IgE results: what are they and when and why to use them? Annals of Allergy Asthma \& Immunology. 2008; 100(6):333-337.10.1016/S1081-1206(10)60595-9

Pace TWW, Heim CM. A short review on the psychoneuroimmunology of posttraumatic stress disorder: From risk factors to medical comorbidities. Brain, Behavior, and Immunity. 2011; 25:613.

Paranjape A, Rask K, Liebschutz J. Utility of STaT for the identification of recent intimate partner violence. Journal of the National Medical Association. 2006; 98(10):1663-1669. [PubMed: 17052059]

Peirce, JM.; Newton, TL.; Buckley, TC.; Keane, TM. Gender and psychophysiology of PTSD. In: Kimerling, R.; Ouimette, P.; Wolfe, J., editors. Gender and PTSD. New York: Guilford; 2002. p. 177-204.

Pfeiffer E. A short portable mental status questionnaire for the assessment of organic brain deficit in elderly patients. Journal of the American Geriatrics Society. 1975; 23(10):433-441. [PubMed: 1159263]

Picotte M, Campbell CG, Thorland WG. Day-to-day variation in plasma interleukin-6 concentrations in older adults. Cytokine. 2009; 47:162-165.10.1016/j.cyto.2009.05.007 [PubMed: 19604707]

Pitiphat W, Garcia RI, Douglass CW, Joshipura KJ. Validation of self-reported oral health measures. Journal of Public Health Dentistry. 2002; 62(2):122-128. [PubMed: 11989207]

Resnick, H. Psychometric review of National Women's Study (NWS) Event History-PTSD Module. In: Stamm, BH., editor. Measurement of Stress, Trauma, and Adaptation. Lutherville, MD: Sidran Press; 1996. p. 214-217. 
Ridker PM. Clinical application of C-reactive protein for cardiovascular disease detection and prevention. Circulation. 2003; 107(3):363-369.10.1161/01.cir.0000053730.47739.3c [PubMed: $12551853]$

Ridker PM, Hennekens CH, Buring JE, Rifai N. C-reactive protein and other markers of inflammation in the prediction of cardiovascular disease in women. New England Journal of Medicine. 2000; 342(12):836-843. [PubMed: 10733371]

Riggs DS, Rothbaum BO, Foa EB. A prospective examination of symptoms of post-traumatic stress disorder in victims of non-sexual assault. Journal of Interpersonal Violence. 1995; 10(2):201-214.

Rose-John S, Scheller J, Elson G, Jones SA. Interleukin-6 biology is coordinated by membrane-bound and soluble receptors: role in inflammation and cancer. Journal of Leukocyte Biology. 2006; 80(2):227-236.10.1189/jlb.1105674 [PubMed: 16707558]

Scheller J, Chalaris A, Schmidt-Arras D, Rose-John S. The pro- and anti-inflammatory properties of the cytokine interleukin-6. Biochimica et Biophysica Acta (BBA) - Molecular Cell Research. 2011; 1813(5):878-888.10.1016/j.bbamcr.2011.01.034

Simmons A, Matthews SC, Stein MB, Paulus MR. Anticipation of emotionally aversive visual stimuli activates right insula. Neuroreport. 2004; 15(14):2261-2265.10.1097/00001756-200410050-00024 [PubMed: 15371746]

Simmons AN, Paulus MP, Thorp SR, Matthews SC, Norman SB, Stein MB. Functional activation and neural networks in women with posttraumatic stress disorder related to intimate partner violence. Biological Psychiatry. 2008; 64:681-690.10.1016/j.biopsych.2008.05.027 [PubMed: 18639236]

Simmons AN, Stein MB, Strigo IA, Arce E, Hitchcock C, Paulus MP. Anxiety positive subjects show altered processing in the anterior insula during anticipation of negative stimuli. Human Brain Mapping. 2011; 32(11):1836-1846.10.1002/hbm.21154 [PubMed: 21181800]

Sjogren E, Leanderson P, Kristenson M, Ernerudh J. Interleukin-6 levels in relation to psychosocial factors: Studies on serum, saliva, and in vitro production by blood mononuclear cells. Brain, Behavior, and Immunity. 2006; 20(3):270-278.10.1016/j.bbi.2005.08.001

Straus, MA.; Hamby, SL.; Warren, WL. The Conflict Tactics Scales Handbook. Los Angeles, CA: Western Psychological Services; 2003.

Sutherland AG, Alexander DA, Hutchison JD. Disturbance of pro-inflammatory cytokines in posttraumatic psychopathology. Cytokine. 2003; 24(5):219-225. [PubMed: 14596818]

Tanda N, Ohyama H, Yamakawa M, Ericsson M, Tsuji T, McBride J, Login GR. IL-1 $\beta$ and IL-6 in mouse parotid acinar cells: characterization of synthesis, storage, and release. American Journal of Physiology - Gastrointestinal and Liver Physiology. 1998; 274(1):G147-G156.

Tjaden, P.; Thoennes, N. Extent, nature, and consequences of intimate partner violence. Washington, D.C: U.S. Department of Justice, National Institute of Justice; 2000.

Tsai J, Pietrzak RH, Southwick SM, Harpaz-Rotem I. Examining the dimensionality of combat-related posttraumatic stress and depressive symptoms in treatment-seeking OEF/OIF/OND veterans. Journal of Affective Disorders. 2011; 135(1-3):310-314.10.1016/j.jad.2011.06.057 [PubMed: 21782249]

Vidovic A, Gotovac K, Vilibic M, Sabioncello A, Jovanovic T, Rabatic S, Dekaris D. Repeated assessments of endocrine- and immune-related changes in posttraumatic stress disorder. Neuroimmunomodulation. 2011; 18(4):199-211.10.1159/000322869 [PubMed: 21335985]

von Känel R, Begre S, Abbas CC, Saner H, Gander ML, Schmid JP. Inflammatory biomarkers in patients with posttraumatic stress disorder caused by myocardial infarction and the role of depressive symptoms. Neuroimmunomodulation. 2010; 17:39-46.10.1159/000243084 [PubMed: 19816056]

Watson D, Clark LA, Tellegen A. Development and validation of brief measures of positive and negative affect: The PANAS scales. Journal of Personality and Social Psychology. 1988; 54(6): 1063-1070. [PubMed: 3397865]

Wong CM. Posttraumatic stress disorder: Advances in psychoneuroimmunology. Psychiatric Clinics of North America. 2002; 25(2):369-383. [PubMed: 12136505] 


\section{Highlights}

- Apparently persistent PTSD differs from remitted PTSD, and from trauma alone.

- In persistent PTSD, salivary IL-6 appears sensitive to anticipatory states.

- In persistent PTSD, salivary IL-6 correlates positively with negative emotional state.

- Soluble IL-6 receptor levels are higher in persistent, compared to remitted, PTSD. 

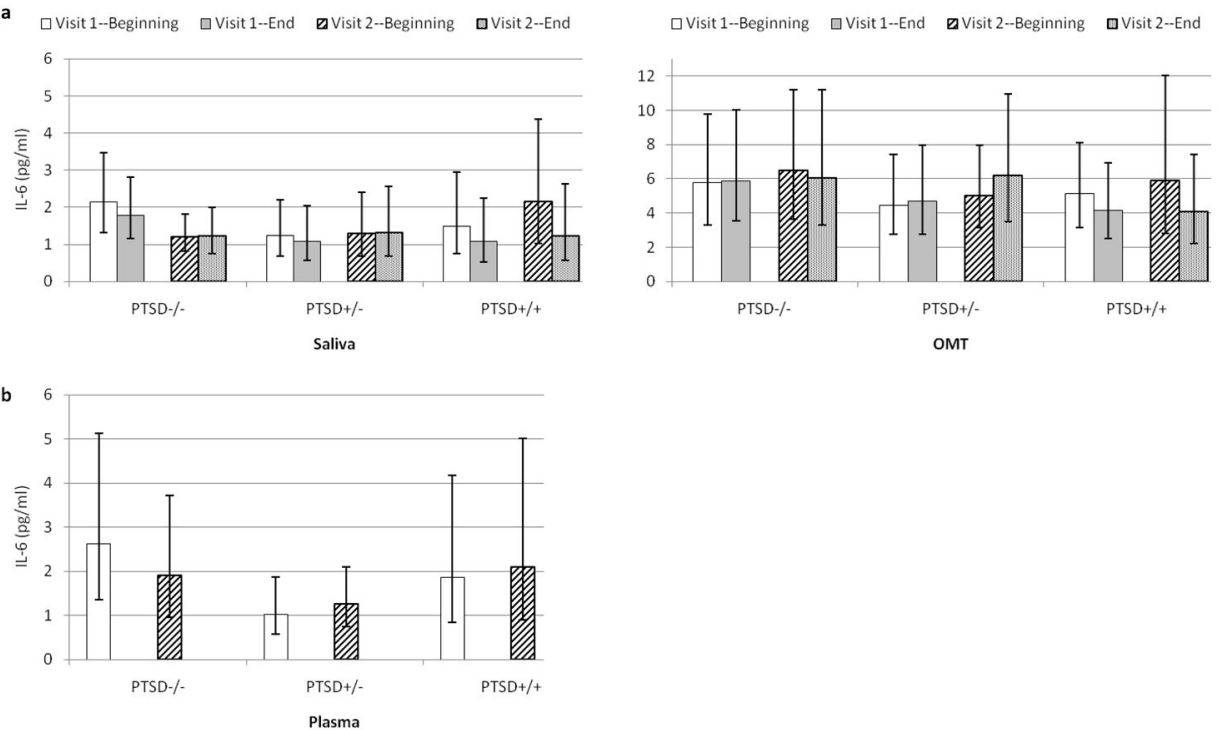

Figure 1.

a-b. Predicted IL-6 Levels by PTSD Group, Visit, and Sample

Note. 1a-Saliva. Averaged across both visits, the PTSD+/+ group showed higher salivary IL-6 levels at the beginning of visits compared with the end $(p=.022)$. Visit $1=$ baseline; Visit 2 = trauma assessment. Error bars depict $95 \%$ CIs corresponding to geometric means. OMT $=$ oral mucosal transudate. 


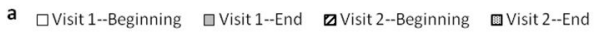

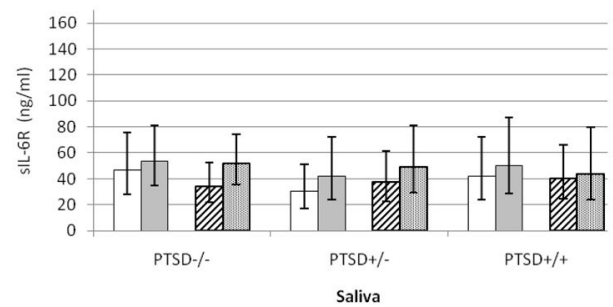

Saliva

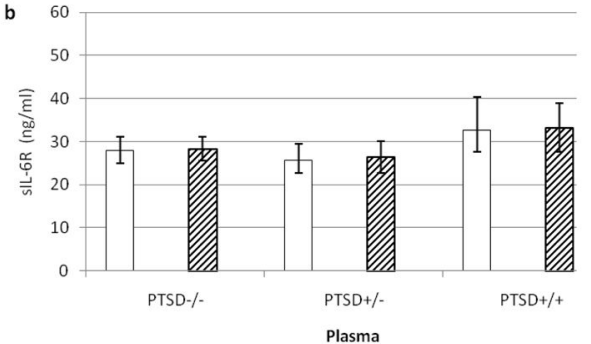

口Visit 1--Beginning घVisit 1--End @Visit 2--Beginning घVisit 2--End

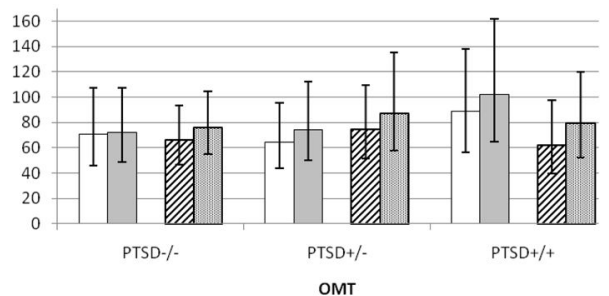

Figure 2.

a-b. Predicted sIL-6R Levels by PTSD Group, Visit, and Sample

Note. 2a-Saliva and OMT. Averaged across all groups and visits, sIL-6R levels were higher at the end of visits than at the beginning ( $p$ s $\leq .0023)$. 2b. sIL-6R levels were higher in the PTSD+/+ group compared to the PTSD $+/$ - group $(p=.05)$. Visit $1=$ baseline; Visit 2 = trauma assessment. Error bars depict 95\% CIs corresponding to geometric means (saliva, OMT) or standard errors (plasma). OMT $=$ oral mucosal transudate. 


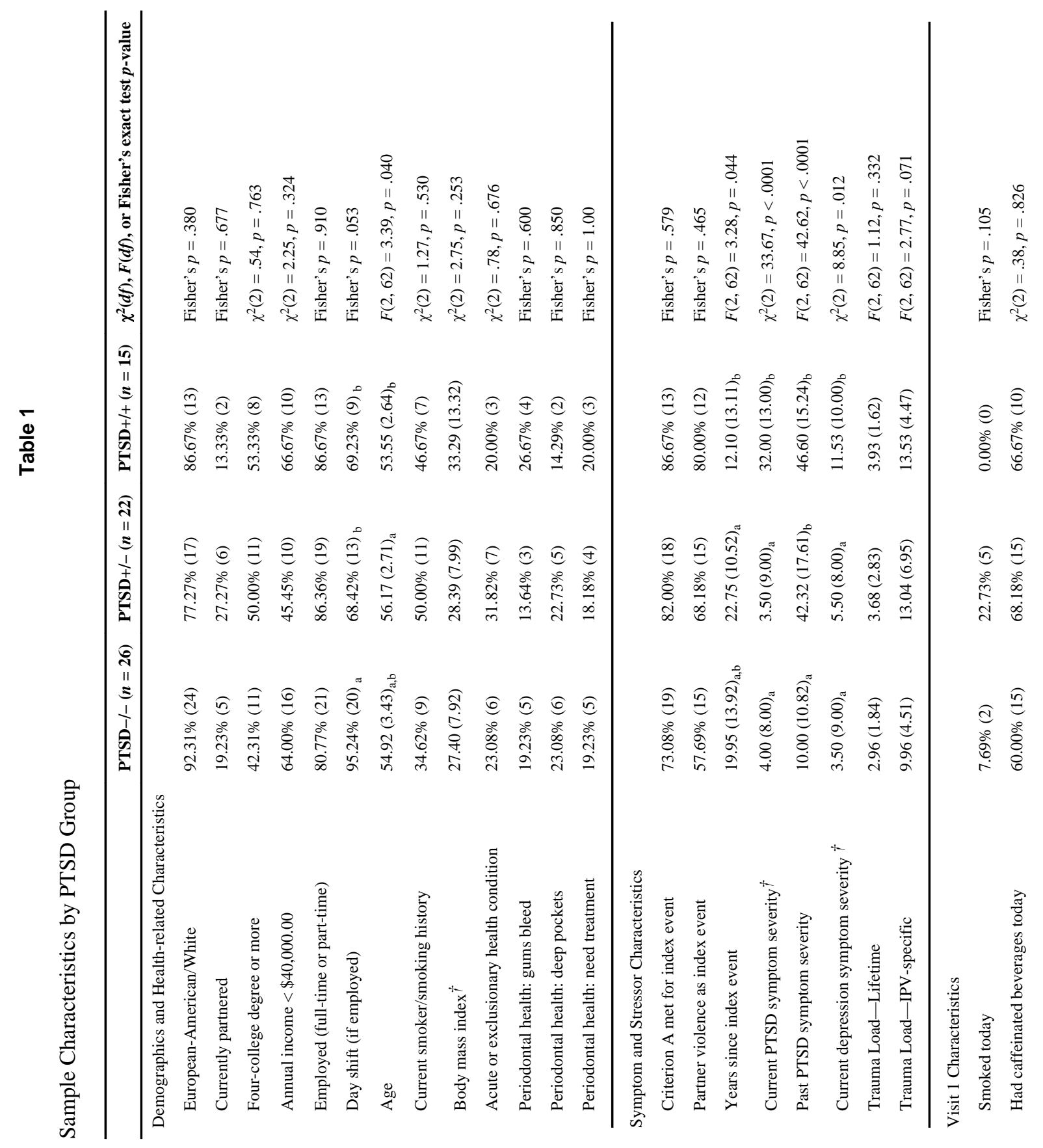



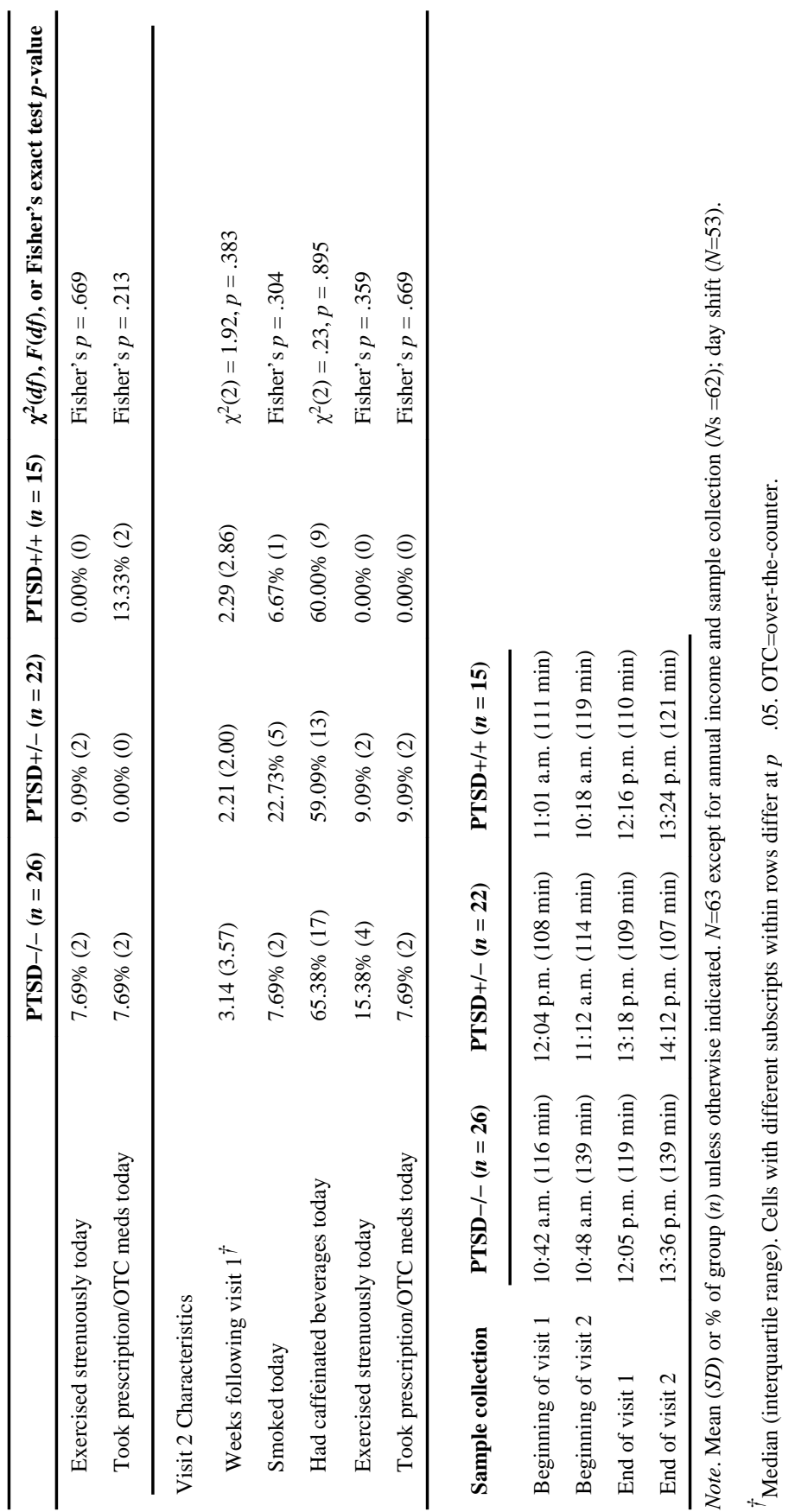

Biol Psychol. Author manuscript; available in PMC 2015 May 01. 